\title{
Estructuras, sistemas modelo y aplicabilidad empírica"
}

\section{Structures, Model Systems, and Empirical Applicability}

\author{
Adolfo García de la Sienra
}

\begin{abstract}
Resumen
Aceptando la distinción que hace Frigg (2010) entre sistemas modelo y estructuras conjuntistas, y reconociendo que los sistemas modelo son una parte importante del aparato de las ciencias empíricas, mi propósito en este artículo es precisamente responder las siguientes preguntas: ¿De qué manera se utilizan las estructuras conjuntistas para hacer aserciones empíricas acerca de sistemas objetivo reales y cuál es el papel en esta tarea de los sistemas modelo y de las estructuras de datos? En particular, ¿de qué manera los sistemas modelo representan-t (para usar la terminología de Frigg) a sus respectivos sistemas objetivo?
\end{abstract}

Palabras clave: estructura conjuntista - sistema modelo - sistema objetivo - aplicación empírica estructuras de datos - estructuralismo

\begin{abstract}
Accepting Frigg's (2010) distinction between model systems and set-theoretical structures, and acknowledging that model systems are an important part of the apparatus of empirical sciences, my purpose in this paper is precisely to respond the following questions: How are set-theoretical structures used to make empirical claims about real objective systems and what is the role in this endeavor of model systems and structures of data? In particular, how do model systems t-represent (to use Frigg's terminology) their respective objective systems?
\end{abstract}

Keywords: set-theoretical structure - model system - objective system - empirical application structures of data - structuralism

\section{Introducción}

Frigg (2010) enfatiza la necesidad de distinguir lo que él llama 'sistemas modelo' (model systems) de las estructuras conjuntistas. Refiriéndose a la concepción que identifica a los sistemas modelo con estructuras conjuntistas, concepción cuyo origen atribuye a Suppes (1960), Frigg sostiene que:

* Recibido: 21 Diciembre 2010. Aceptado en versión revisada: 26 Marzo 2011.

+ Instituto de Filosofía/Facultad de Economía, Universidad Veracruzana. Para contactar al autor, por favor escriba a: asienrag@gmail.com.

¥Este trabajo participa del proyecto de investigación PICTR 2006 № 2007, de la Agencia Nacional de Promoción Cientifica y Tecnológica, de la República Argentina.

Metatheoria 1(2)(2011): 29-37. ISSN 1853-2322.

(C) Editorial de la Universidad Nacional de Tres de Febrero. Publicado en la República Argentina. 
This conception of model systems is too narrow. Although structures do play an important role in scientific modelling (I come back to this below), model systems cannot be identified with structures. What is missing in the structuralist conception is an analysis of the physical character of model systems. The view of model systems that I advocate regards them as imagined physical systems, i.e. as hypothetical entities that, as a matter of fact, do not exist spatio-temporally but are nevertheless not purely mathematical or structural in that they would be physical things if they were real. If the Newtonian model system of sun and earth were real, it would consist of two spherical bodies with mass and other concrete properties such as hardness and colour, properties that structures do not have; likewise, the populations in the Lotka-Volterra model would consist of flesh-and-blood animals if they were real, and the agents in Edgeworth's economic model would be rational human beings (Frigg 2010, p. 253).

Frigg considera que hay dos razones para preferir su concepción a la estructuralista:

The first is that scientists often talk about model systems as if they were physical things, which is a natural thing to do if models are imagined physical entities. [...] The second reason has to do with how model systems relate to the world. A structure is not about anything in the world, let alone about a particular target system. Those who take model systems to be structures suggest connecting structures to target systems by setting up a morphism between them (the most common morphism is isomorphism; other suggestions include partial isomorphism, homomorphism, and embedding). But a morphism holds between two structures and not between a structure and a part of the world per se. In order to make sense of the notion that there is a morphism between a model system and its target we have to assume that the target exemplifies a particular structure, and this cannot be had without bringing nonstructural features into play (Frigg 2010, pp. 253-254).

Frigg observa que "in order for it to be true that a target system possesses a particular structure, a more concrete description must be true of the system as well" (Frigg 2010, p. 254), porque los "structural claims are abstract in the sense that they cannot be true unless some more concrete claims are true as well" (Frigg 2010, p. 254) y acepta que: "[...] this by itself would not have to worry the structuralist. The problem, and this is the second step, arises when we realise that the descriptions we choose to ground structural claims are almost never true descriptions of the target system" (Frigg 2010, p. 254).

Y agrega:

Taken literally, descriptions that ground structural claims (almost always) fail to be descriptions of the intended target system. Instead, they describe a hypothetical system distinct from the target system. This has unfortunate consequences for the structuralist. If the descriptions employed to attribute a structure to a target system were just plain descriptions of that system, then the claim that model systems are just structures would appear at least prima facie plausible. But once we acknowledge that these descriptions describe hypothetical systems rather than real target systems, we also have to acknowledge that hypothetical systems are an important part of the theoretical apparatus we employ, and that they therefore have to be included in our analysis of how scientific modelling works. (Frigg 2010, pp. 254-255) 
Aceptando la distinción entre sistemas modelo y estructuras conjuntistas, y reconociendo que los sistemas modelo son una parte importante del aparato de las ciencias empíricas, mi propósito en este artículo es precisamente resolver este problema. ¿De qué manera se utilizan las estructuras modelo para hacer aserciones empíricas acerca de sistemas objetivo reales y cuál es el papel en esta tarea de los sistemas modelo y de los modelos de datos? En particular, ¿de qué manera los sistemas modelo representan-t (para usar la terminología de Frigg) a sus respectivos sistemas objetivo? En la siguiente sección trataré esta última pregunta. Dedicaré la última al problema de la cuádruple relación estructura-sistema modelo-sistema objetivo-modelo de datos y a la cuestión de cómo es posible aplicar teorías empíricas a sistemas objetivo a través de sistemas modelo.

\section{La representación-t de sistemas objetivo}

En su interesante teoría de la pretensión, Frigg distingue las representaciones-p, las cuales son representaciones de objetos meramente ficticios, de las representaciones-t, las cuales además representan un cierto sistema objetivo:

These two senses of 'representation' need to be clearly distinguished, and for this reason I call the former 'p-representation' ('p' for 'prop') and the latter 't-representation' (' $t$ ' for 'target'). Using this idiom, the two acts mentioned in the introduction can be described as, first, introducing a p-representation specifying a hypothetical object and, second, claiming that this imagined object t-represents the relevant target system (Frigg 2010, p. 264).

Frigg cree que "The apparent comparison with a nonexistent object eventually comes down the unproblematic comparison of properties, and the statement making this comparison is true if the statement comparing the properties with each other is true" (Frigg 2010, pp. 263-264).

Quisiera discutir con cierta precisión, mediante un ejemplo importante en teoría económica, cómo se suele y se debe hacer esta comparación.

En efecto, es usual en el proceso de elaboración de teorías científicas la construcción de sistemas modelo a partir de conceptos que, si bien se originan en la experiencia y corresponden a propiedades reales de entes reales, han sufrido una suerte de deformación. Por ejemplo, es indudable que los agentes económicos reales son seres humanos que manejan cierta información, que pueden recordar algunos eventos y que, además, pueden realizar operaciones aritméticas. Es por ello que los predicados

$$
\begin{aligned}
& \text { 'x posee información' } \\
& x \text { tiene memoria' }
\end{aligned}
$$

y

$$
\text { ' } x \text { es capaz de realizar cálculos' }
$$

son verdaderos de los seres humanos que realizan transacciones económicas en cualquier lugar. Sin embargo, si bien la teoría económica tiene que referirse de alguna manera a las propiedades que expresan, el uso del método matemático re- 
quiere el uso de versiones deformadas de tales predicados. Algunas especializaciones de la teoría económica requieren, por ejemplo, que los agentes económicos posean información perfecta, memoria perfecta y poderes computacionales ilimitados. Así, por ejemplo, los predicados (1), (2) y (3) son deformados para obtener

$$
\begin{aligned}
& \text { ' } x \text { posee información perfecta' } \\
& \text { ' } x \text { tiene memoria perfecta' }
\end{aligned}
$$

y

$$
\text { ' } x \text { tiene poderes computacionales ilimitados' }
$$

La conjunción de predicados

' $x$ posee información perfecta $\wedge x$ tiene memoria perfecta $\wedge x$ tiene poderes computacionales ilimitados' (7)

define un predicado compuesto $P(x)$ que sirve como "prop" para generar un objeto ficticio -un sistema modelo-, inexistente en la realidad, pero necesario para encauzar el razonamiento matemático. Como lo dijera Walras:
el método matemático no es un método experimental; es un método racional [...] la ciencia pura de la economía debería entonces abstraer y definir concep- tos de tipos ideales en términos de los cuales conducir su razonamiento. El re- torno a la realidad no debería tener lugar hasta que la ciencia sea completada y entonces solamente con vista en las aplicaciones prácticas (Walras 1954, p. 71). (La traducción es mía.)

Llamaremos 'conceptos idealizados' (o, brevemente, 'idealizaciones') a los conceptos ideales obtenidos por deformación a partir de conceptos verdaderamente predicables de entes reales si, a pesar de esta deformación, estos son significativos -aunque sean falsos- de estos objetos reales y conservan una intensión emparentada con aquellos. Siguiendo a Frigg (2010, p. 252), llamo 'sistema modelo' a los tipos ideales construidos mediante conjunciones de predicados que expresan idealizaciones. Los sistemas modelo representan-t a sus respectivos sistemas objetivo en primer lugar porque los predicados que definen a los primeros son deformaciones inteligibles de predicados literalmente verdaderos de los segundos. Pero esta representación es hasta cierto punto convencional y obedece a que, usualmente, el sistema modelo fue construido precisamente con la intención de representar ese sistema objetivo, la comunidad científica relevante así lo estima, y es así como se presenta en los libros de texto de la disciplina. Si el sistema modelo $m$ representa-t al sistema objetivo $r$ escribimos $m \triangleq r$, siguiendo una notación introducida por Bunge (1972).

\section{Sistemas modelo y estructuras}

Los axiomas de una teoría económica consistente definen directamente un predicado conjuntista (como $\mathfrak{A}$ es una 'estructura de preferencia regular') pero también son oblicuamente verdaderos, al menos bajo alguna interpretación típica de los términos primitivos, de sistemas modelo. Considérese la siguiente definición de un predicado conjuntista. 
Definición 1: $\mathfrak{A}$ es una estructura de preferencia regular syss existen $X \mathrm{y} \succsim$ tales que

(0) $\mathfrak{A}=\langle X, \succsim\rangle$

(1) $X$ es un conjunto no vacío

(2) $\succsim$ es una relación binaria sobre $X$

(3) $\succsim$ es conectada

(4) $\succsim$ es transitiva.

Es discutible que la relación de preferencia exhibida (parcialmente) por un agente humano en un momento dado sea conectada y transitiva. Sin embargo, el desarrollo sistemático de la teoría puede proceder como si esta fuese acerca de un agente que satisface con precisión los axiomas (3) y (4).

La teoría de la utilidad ha procedido a través de la construcción de sistemas modelo dotados de propiedades idealizadas. Se ha supuesto, por ejemplo, que el agente (un sistema modelo), además de satisfacer el predicado $P(x)$ definido arriba en (7), define su relación de preferencia sobre un espacio de cestas de consumo que es representable por un subconjunto convexo del ortante no negativo de $\Omega$ de $\mathbb{R}^{L}$ (otro sistema modelo). Otras idealizaciones que han sido introducidas sobre estas son las de relación de preferencia continua o relación de preferencia insaciada (o localmente insaciada).

$\mathrm{Al}$ ser idealizaciones, los predicados "relación de preferencia continua" o "relación de preferencia insaciada" son deformaciones (inteligibles) de predicados que denotan propiedades reales. Podemos imaginar y entender perfectamente bien que un pequeño (inobservable) cambio en un menú de consumo no debería alterar notablemente (observablemente) la satisfacción que el primero aporta a un consumidor real. Igualmente, dentro de ciertos límites, al menos, podemos entender que un consumidor prefiera más bienes que menos. En todo caso, las idealizaciones en cuestión son falsas de los consumidores que la teoría ha focalizado como sistemas objetivo pero entonces, si son falsas, es que siguen teniendo un significado inteligible. Todas las idealizaciones anteriormente mencionadas (espacio de consumo, relación de preferencia regular, continua, insaciada) son necesarias para demostrar la existencia de funciones de utilidad que posean ciertas características requeridas por la teoría económica (continuidad, monotonía).

En este ejemplo se ve que un predicado conjuntista (bajo cierta interpretación de los términos primitivos) es satisfecho con toda exactitud por uno o varios sistemas modelo que a su vez representan ciertos sistemas objetivos. Es un error categorial decir que un predicado conjuntista es satisfecho por un sistema objetivo, pues los sistemas objetivos no son entidades conjuntistas. Así (dejando de lado el problema de Ramsey) no es posible expresar la aserción empírica de una teoría $T$ diciendo que los sistemas objetivo (reales) en una cierta clase satisfacen el predicado conjuntista que define las estructuras de la teoría.

Una forma más correcta de expresar la aserción empírica consistiría, pues, en afirmar que la teoría $T$ es aplicable a la clase de sistemas objetivo $C$. Para hacer plausible esta aserción, sin embargo, se requiere hacer ciencia normal aplicando efectivamente la teoría a cada uno de los casos en $C$. La aplicación a un caso pretendido $c \in C$ consiste típicamente en elaborar un objeto modelo $m$ que satisface 
los términos no teóricos de $T$ (bajo cierta interpretación semántica), y tal que $m$ $\triangleq c$. Esto resuelve el problema de que las descripciones que escogemos para aterrizar aserciones estructuralistas casi nunca son descripciones verdaderas del sistema objetivo (Frigg 2010, p. 254). Desde luego, así descrita, la aserción empírica involucra aserciones verdaderas que no son expresables en términos puramente de estructuras. Pero la verdad es que nadie ha sostenido que lo sean: el estructuralismo siempre ha insistido en que dichas aserciones involucran consideraciones históricas y pragmáticas. Incluso Suppes, al defender su idea de medición fundamental, insiste en que las "estructuras empíricas" son el resultado de consideraciones no sistemáticas.

\section{Sistemas modelo y sistemas objetivo}

Para terminar este artículo, quisiera mostrar mediante un ejemplo de qué manera se entrelazan las estructuras con los sistemas modelo y con los sistemas objetivo. Consideraremos la teoría clásica de la demanda.

La teoría clásica de la preferencia (si se me permite llamarla así) empieza por definir el predicado "estructura de preferencia clásica", del modo siguiente.

Definición 2: $\mathfrak{A}$ es una estructura de preferencia clásica syss existen $X \mathrm{y} \succsim$ tales que

(0) $\mathfrak{A}=\langle X, \mathfrak{B}, \succsim\rangle$

(1) $X$ es un subconjunto del ortante no negativo del espacio euclidiano $\mathbb{R}^{L}$

(2) $\langle X, \succsim\rangle$ es una estructura de preferencia regular

(3) $\mathfrak{B}$ es una familia de subconjuntos compactos de $X$

(4) $\succsim$ es estrictamente convexa

(5) $\succsim$ es continuamente diferenciable.

Es posible demostrar que la relación de preferencia $\succsim$ es representable mediante una función de utilidad $u: X \rightarrow \mathbb{R}$ que es cuasicóncava y continuamente diferenciable, de modo que $u$ asume un máximo exactamente en un punto $x_{B}{ }^{*} \in B$, para cada $B \in \mathfrak{B}$. La función $u: \mathfrak{B} \rightarrow X$ que asigna a cada $B \in \mathfrak{B}$ el punto $x_{B}{ }^{*} \in B$ es la función de demanda walrasiana.

La teoría clásica de la demanda requiere la construcción de un sistema modelo $m$ consistente en un consumidor con memoria infinita que sabe de antemano cuál sería su elección al confrontar las opciones de cualquier conjunto $B \in \mathfrak{B} .{ }^{1}$ Esta información es representada en una estructura conjuntista mediante una función de demanda "no teórica" (relativamente a la teoría $T$ de la demanda clásica) $\eta: \mathfrak{B} \rightarrow X$. Si la matriz de Slutsky correspondiente a $\eta$ es semidefinida negativa y simétrica, el consumidor sabe que coincide con una función de demanda walrasiana $\mu \mathrm{y}$, además, dado que $m$ posee capacidades computacionales ilimitadas, puede determinar instantáneamente la relación de preferencia de la que proviene dicha $\mu$ (y por lo tanto la $\eta$ ).

\footnotetext{
${ }^{1}$ Los conjuntos $B$ en este caso son los conjuntos presupuestales definidos como $\{x \in X / \mathbf{p x} \leq w\}$, donde $\mathbf{p}$ es un sistema de precios y $w$ un nivel de renta. El conjunto de los precios es $P$ y el de las rentas es $W$.
} 
Si c es un sistema objetivo (un consumidor humano de carne y hueso) y la comunidad científica representa a $c$ con $m$, el científico normal puede intentar aplicar la teoría $T$ a $c$. Que $T$ sea aplicable o no aplicable a $c$ es una cuestión enteramente empírica. Decir que lo es, es un claim que tiene que ser sustentado con base en datos empíricos. Varian (1983) ha subrayado que hay dos aproximaciones a este problema. Uno, el enfoque basado en el cálculo diferencial e integral, el cual se originó en el trabajo de Slutsky (1915) y Antonelli (1886), deriva condiciones necesarias y suficientes sobre las derivadas de la función de demanda $\eta$. El segundo, que se origina en el trabajo de Samuelson (1938, 1947, 1948), deriva condiciones sobre la función de demanda implicadas por la conducta maximizadora y es llamado 'no-paramétrico' porque no supone forma específica alguna para la función $\eta$.

The distinction between the two approaches is very important in empirical work. The calculus approach assumes the entire demand function [nuestra $\eta$ ] is available for analysis, while the algebraic approach assumes only a finite number of observations on consumer behaviour is available. Since all existing data on consumer behavior does consist of finite number of observations, the latter assumption is much more realistic (Varian 1983, p. 99).

\begin{tabular}{|c|c|}
\hline Argumento & Valor de $\hat{\eta}$ \\
\hline$\left(\mathbf{p}^{1}, w^{1}\right)$ & $\hat{\mathbf{x}}^{1}=\hat{\eta}\left(\mathbf{p}^{1}, w^{1}\right)$ \\
\hline$\left(\mathbf{p}^{2}, w^{1}\right)$ & $\hat{\mathbf{x}}^{2}=\hat{\eta}\left(\mathbf{p}^{2}, w^{2}\right)$ \\
\hline$\ldots$ & $\ldots$ \\
\hline$\left(\mathbf{p}^{k}, w^{k}\right)$ & $\hat{\mathbf{x}}^{n}=\hat{\eta}\left(\mathbf{p}^{k}, w^{k}\right)$ \\
\hline$\ldots$ & $\ldots$ \\
\hline$\left(\mathbf{p}^{N}, w^{N}\right)$ & $\hat{\mathbf{x}}^{N}=\hat{\eta}\left(\mathbf{p}^{N}, w^{N}\right)$ \\
\hline
\end{tabular}

Podemos explicar el método no paramétrico mediante el concepto de estructura de datos. A partir de la observación de la conducta del agente obtenemos una estructura de datos:

$$
\hat{\mathfrak{D}}=\langle X, F, \hat{\eta}\rangle
$$

donde $\hat{\eta}$ es una función definida sobre un subconjunto finito $F$ de $P \times W$, precisamente el de pares de sistemas de precios y rentas bajo las cuales se ha observado la conducta del consumidor. La función de demanda empírica $\hat{\eta}$ es obviamente finita y discreta, por lo que la estructura $\mathfrak{D}$ se puede exponer mediante la tabla. El método no paramétrico no busca construir directamente la completa función de demanda $\eta$, sino que utiliza un algoritmo para construir directamente una función de utilidad que "racionalice" los datos, siempre y cuando la estructura $\mathfrak{D}$ satisfaga las condiciones del siguiente teorema.

TeOrema. (Afriat) Las siguientes condiciones son equivalentes:

(1) Existe una función de utilidad no saciada que racionaliza los datos.

(2) Los datos satisfacen el axioma general de la preferencia revelada. 
(3) Existen números $U^{i}, \lambda^{i}>0(i=1, \ldots, N)$ que satisfacen las desigualdades de Afriat:

$U^{i} \leq U^{j}+\lambda^{j} \mathbf{p}^{j}\left(\mathbf{x}^{i}-\mathbf{x}^{j}\right)$

para $i, j=1, \ldots, N$.

(4) Existe una función de utilidad cóncava, monótona, continua y no saciada que racionaliza los datos. ${ }^{2}$

Afriat (1967) y Varian (1982) proveyeron algoritmos mediante los cuales es posible construir una función de utilidad $u$ que racionalice los datos de una estructura $\mathfrak{D}$. Esto significa "brincar", por así decirlo, de un modelo de datos a la función T-teórica $u$. Luego es posible incrustar $\mathfrak{D}$ en la estructura parcial que consiste de la función de demanda walrasiana $\mu$ inducida por $u$. Es decir, $\mu$ restringida a $F$ coincide puntualmente con $\hat{\eta}$. El método paramétrico busca determinar primero la función de demanda no teórica $\eta$ sin involucrar al término teórico $u$ y luego trata de recuperar $u$ a partir de $\eta .^{3}$

Queda elucidada, así, por lo menos en este ejemplo, la cuádruple relación estructura-sistema modelo-sistema objetivo-modelo de datos, y resuelta la cuestión de cómo es posible aplicar teorías empíricas a sistemas objetivo a través de sistemas modelo. El enigma se resuelve a través de la relación $\triangleq$, y de notar que, finalmente, la aplicabilidad de una teoría a un sistema objetivo determinado (representado por sistemas modelo idealizados que satisfacen con precisión los axiomas de la teoría) es una cuestión enteramente empírica.

${ }^{2}$ Para una demostración, véase Varian (1983).

${ }^{3}$ Para una explicación estructuralista de este procedimiento, véase García de la Sienra (2009). 


\section{Bibliografía}

Afriat, S.N. (1967), "The Construction of Utility Functions from Expenditure Data”, International Economic Review 8(1): 67-77.

Antonelli, G.B. ([1886]1971), "On the Mathematical Theory of Political Economy", en Chipman, J.S., Hurwicz, L., Ricther, M.K y H.F. Sonnenschein (eds.), Preferences, Utility, and Demand, Nueva York: Harcourt Brace Jovanovich, 1971, pp. 333-364.

Bunge, M. (1972), Teoría y realidad, Barcelona: Ariel.

Frigg, R. (2010), "Models and Fiction”, Synthese 172(2): 251-268.

García-Bermejo, J.C. (ed.) (2009), Sobre la Economía y sus métodos, Enciclopedia Iberoamericana de Filosofía 30, Madrid: Editorial Trotta.

García de la Sienra, A. (2009), "La aplicación a la Economía de la concepción estructuralista de las teorías”, en García-Bermejo (2009), pp. 355-366.

Samuelson, P. (1938), “A Note on the Pure Theory of Consumer Behavior”, Económica 15: 6171, Vol. 5, pp. 61-71.

Samuelson, P. (1947), Foundations of Economic Analysis, Cambridge, Mass.: Harvard University Press.

Samuelson, P. (1948), "Consumption Theory in Terms of Revealed Preference", Económica 15: 243-253.

Slutsky, E., ([1915]1952), "On the Theory of the Budget of the Consumer", en Stigler, G.J. y K.E. Boulding (eds.), Readings in Price Theory, Chicago: Richard D. Irwin, pp. 27-56.

Suppes, P. (1960), "A Comparison of the Meaning and Uses of Models in Mathematics and the Empirical Sciences", Synthese 12: 287-301.

Varian, H.R. (1982), “The Non-Parametric Approach to Demand analysis", Econometrica 50(4): 945-973.

Varian, H.R. (1983), "Non-Parametric Tests of Consumer Behaviour", Review of Economic Studies 50(1): 99-110.

Walras, L. (1954), Elements of Pure Economics, Londres: Allen and Unwin. 